\title{
Prehospital Pediatric Endotracheal Intubation
}

\author{
Samuel J. Stratton, MD, MPH
}

Pediatric endotracheal intubation has been a controversial subject in paramedic responder based Emergency Medical Services (EMS) systems for more than two decades. Pediatric intubation is difficult in the field, and known to have potentially fatal complications that include esophageal endotracheal tube misplacement and tube dislodgement with loss of airway during transport. In addition, time taken in the field to place a pediatric endotracheal tube can delay transport for more definitive emergency care. Also, the procedure is invasive, with a potential for damage of a child's upper airway and vocal cords. Because the pediatric airway is not as developed as the adult airway, and is smaller, pediatric intubation is recognized to be more difficult and prone to complication than adult intubation. ${ }^{1}$

Despite the challenges and potential for complication with prehospital pediatric intubation, the procedure is considered a standard for EMS practice in the United States. Pediatric intubation equipment is considered a required standard for advanced life support ambulances by the American Academy of Pediatrics, American College of Emergency Physicians, American College of Surgeons, National Association of EMS Physicians, and EMS for Children groups. ${ }^{2}$ Considering the endorsement of these organizations, it would seem that prehospital pediatric intubation has obvious science-based support for the procedure being a U.S. national standard.

Oddly, scientific support for prehospital pediatric intubation does not exist. In 1989, Losek et al and Aijian et al published separate descriptive studies that explored the unique characteristics of prehospital pediatric intubation. They reported that successful placement of pediatric endotracheal tubes in the field was less than for adult intubation, and the procedure was made difficult by problems in visualization of the immature pediatric airway landmarks, inappropriate field use of small endotracheal tubes, and accidental extubation during transport. ${ }^{3,4}$ Despite recognizing the differences between prehospital pediatric and adult intubation, prehospital pediatric along with adult intubation became a standard for EMS systems providing advanced life support. In a sense it appeared that during the 1980s there was little understanding among EMS Medical Directors and educators of pediatric intubation being a different process from that of adult intubation. Eleven years after the 1989 studies of Losek and Aijian, Gausche et al published a randomized, clinical trial that compared prehospital pediatric endotracheal intubation with bag-valve-mask ventilation. ${ }^{5}$ This trial showed, in rapid transport urban EMS systems, that there was no advantage for prehospital pediatric intubation over the basic life support procedure of bag-valve-mask ventilation. The Gausche trial study is criticized for use of intention-to-treat design with possible preference for bagvalve-mask ventilation of patients assigned to the intubation arm. But the trial study, as opposed to previous and many subsequent prehospital pediatric intubation studies, used the outcome of interest of survival to hospital discharge with good neurological performance. While papers on the subject of prehospital pediatric intubation have been published since 2000, none that have used similar outcome results have refuted the findings of the Gausche paper.

Of course, the research referred to above is specific to paramedic-based EMS systems (Anglo-American Model) and cannot be extended to EMS response models that include physician or nurse responders (Franco-German Model). But, the same lack of scientific support for prehospital pediatric intubation exists for the Franco-German Model. ${ }^{6}$ While pediatric intubation success rates are higher for physician-response EMS systems, 7 a study of intubation versus basic airway management techniques has not been done. At present, Franco-German style EMS systems are beginning to explore intubation and it has been found that uniform data to support intubation 
studies, either pediatric or adult, does not exist at present.(7) In fact, a 2008 Cochrane Review of prehospital intubation that included review of all global studies and reports concluded that "In trauma and paediatric patients, the current evidence base provides no imperative to extend the practice of prehospital intubation in urban systems." 8

As it now stands, pediatric intubation appears to be a "standard" prehospital procedure practiced world-wide in advancedlife support EMS systems. This practice persists despite lack

\section{References}

1. Perkin R, van Stralen D, Mellick LB. Managing pediatric airway emergencies: anatomic considerations, alternative airway and ventilation techniques, and current treatment options. Pediatr Emerg Rep. 1996;1(1):1-12.

2. Policy Statement-Equipment for Ambulances. Pediatrics. 2009;124(1):e166-e171.

3. Losek JD, Bonadio WA, Walsh-Kelly C, Hermes H, Smith D, Glaeser PW. Prehospital pediatric endotracheal intubation performance review. Pediatric Emerg Care. 1989;5(1):1-4.

4. Aijian P, Tsai A, Knopp R, Kallsen GW. Endotracheal intubation of pediatric patients by paramedics. Ann Emerg Med. 1989;18(5):489-494.

5. Gausche M, Lewis RJ, Stratton SJ, et al. Effect of out-of-hospital pediatric endotracheal intubation on survival and neurological outcome. JAMA. 2000;283(6):783-790. of evidence to support the procedure and the existence of potentially fatal complications. It seems that EMS medical directors, researchers, and educators should avoid considering prehospital pediatric intubation an evidence-based standard, when the evidence does not exist. As important is the immediate need to design and conduct quality studies to further explore the benefits and risks that may or may not establish pediatric intubation as a standard of practice for advanced life support EMS systems.

6. Timmermann A, Russo SG, Hollmann MW. Paramedic versus emergency physician emergency medical service: role of the anaesthesiologist and the European versus the Anglo-American concept. Curr Opin Anaesthesiol. 2008:21(2):222-227.

7. Eich C, Roessler M, Nemeth M, Russo SG, Heuer JF, Timmermann A. Characteristics and outcome of prehospital paediatric tracheal intubation attended by anaesthesia-trained emergency physicians. Resuscitation. 2009;80(12):13711377.

8. Lecky F, Bryden D, Little R, Tong N, Moulton C. Emergency intubation for acutely ill and injured patients. Cochrane Database Syst Rev. 2008;16(2):CD001429. 